\title{
Inversion formula for the internucleus potential using sub-barrier fusion cross sections
}

\author{
A. B. Balantekin \\ Center for Theoretical Physics, Laboratory for Nuclear Science, Massachusetts Institute of Technology, \\ Cambridge, Massachusetts 02139 \\ S. E. Koonin \\ Kellogg Radiation Laboratory, California Institute of Technology, Pasadena, California 91125 \\ J. W. Negele \\ Center for Theoretical Physics, Laboratory for Nuclear Science and Department of Physics, \\ Massachusetts Institute of Technology, Cambridge, Massachusetts 02139
}

(Received 6 June 1983)

\begin{abstract}
Subject to the assumption that sub-barrier fusion is described by an effective one-dimensional energy-independent local potential barrier, the Jeffreys-Wentzel-Kramers-Brillouin approximation for the fusion cross sections is inverted to determine this effective potential. Potential barriers, with their associated experimental errors, are presented for ${ }^{13} \mathrm{C}+{ }^{13} \mathrm{C}$ and ${ }^{40} \mathrm{Ca}+{ }^{40} \mathrm{Ca}$, and the assumption of a local effective potential is shown to be inadequate for ${ }^{64} \mathrm{Ni}+{ }^{64} \mathrm{Ni}$.
\end{abstract}

$\left[\begin{array}{c}\text { NUCLEAR REACTIONS Inversion formula relating fusion cross sections to } \\ \text { ion-ion potential. }\end{array}\right]$

Cross sections for the fusion of two nuclei at sub-barrier energies are of interest both for what they reveal about the nucleus-nucleus interaction and for their astrophysical importance. The traditional approach to the interpretation of such cross sections is to assume that fusion is governed by the penetration of a one-dimensional energy-independent local potential barrier, to describe its height, location, and shape in terms of a few parameters, and then to vary these to fit the cross sections. The systematics of internucleus potentials obtained in this way have been discussed in Ref. 1. However, several recent measurements of fusion cross sections show significant deviation from the behavior expected. These data included unexpected excesses in the sub-barrier fusion of intermediate-mass systems $^{2}$ and the nonsystematic behavior of systems involving two $p$-shell nuclei. ${ }^{3}$ Whether such discrepancies are due to the inadequacies of the parametrization used, ${ }^{4}$ the structure of the particular nuclei involved, ${ }^{5}$ the quantum fluctuations of the nuclear surfaces, ${ }^{6,7}$ the multidimensional dynamics of the fusion process, ${ }^{8}$ or some combination of these effects remains an interesting, but unanswered, question.

In this paper, we present a simple framework for the analysis of sub-barrier fusion data which may help to clarify the situation. It is based on the Jeffreys-WentzelKramers-Brillouin (JWKB) approximation, and allows an effective one-dimensional potential barrier to be determined directly from the experimental data. This barrier can then be used to determine the validity of phenomenological potentials or, should it be pathological, the validity of the one-dimensional barrier-penetration picture itself.

We assume that the effective potential between the two ions, $V$, as a function of the separation of their centers of mass, $r$, has a single quadratic maximum at a barrier radius $R_{B}$ of height $V\left(R_{B}\right)=B$ and that inside the barrier complete absorption into the fusion channel takes place.
The fusion cross section is given by a sum over all partial waves

$$
\sigma=\frac{\pi \hbar^{2}}{2 m E} \sum_{L=0}^{\infty}(2 L+1) T_{L}(E),
$$

where $E$ is the center-of-mass energy and $m$ the reduced mass. (In the spirit of our semiclassical treatment, we ignore symmetry constraints which might restrict the sum to even or odd $L$ only.)

At energies $E<B$, we adopt the JWKB form of the penetration coefficients ${ }^{9} T_{L}(E)$, as extended by Hill and Wheeler ${ }^{10}$ to the case of barriers with a quadratic maximum

$$
\begin{aligned}
& T_{L}(E)=\left(1+e^{2 S_{L}(E)}\right)^{-1}, \\
& S_{L}(E)=\int_{r_{1}}^{r_{2}}\left[\frac{2 m}{\hbar^{2}}[V(r)-E]+\frac{L(L+1)}{r^{2}}\right]^{1 / 2} d r .
\end{aligned}
$$

The limits of the "action" integral $\left(r_{1}<r_{2}\right)$ are determined by the zeros of the integrand. (We assume that there are only two such turning points.)

To obtain tractable expressions, we suppose that, at a given $E$, the $L$ dependence of $T_{L}(E)$ can be reproduced by simply shifting the energy. That is,

$$
T_{L}(E) \approx T_{0}\left[E-\frac{L(L+1) \hbar^{2}}{2 m R^{2}(E)}\right],
$$

where $R(E)$ characterizes an effective moment of inertia. This amounts to assuming that the centrifugal term in the integrand of (2b) is independent of $r$. To study the validity of this approximation we compared two sets of cross sections from the phenomenological potential of Krappe, Nix, and Sierk [Eqs. (17)-(20) of Ref. 11, hereafter denot- 
ed the KNS potential] for the ${ }^{64} \mathrm{Ni}+{ }^{64} \mathrm{Ni}$ system. In one set Eq. (2b) was used and for the other set the term $L(L+1) / r^{2}$ was replaced by $L(L+1) / r_{B}^{2}$, where $r_{B}$ is the actual position of the barrier of the KNS potential. These cross sections differed by less than $10 \%$, which is smaller than realistic experimental uncertainties. The validity of our assumption (3) in cases of physical interest will be demonstrated further below.

If large values of $L$ are important in the sum (1a), we can approximate it by an integral over $L$, and, using (3) obtain

$$
E \sigma(E)=\pi R^{2}(E) \int_{-\infty}^{E} d E^{\prime} T_{0}\left(E^{\prime}\right)
$$

Upon using (2a) to relate $T_{0}$ and $S_{0}$, we find

$$
S_{0}(E)=\frac{1}{2} \log \left\{\left[\frac{d}{d E}\left[\frac{E \sigma}{\pi R^{2}}\right]\right]^{-1}-1\right\} .
$$

Thus, if $R(E)$ is specified (see below), $S_{0}(E)$ is completely determined by the experimental data, $\sigma(E)$. Furthermore, $B$ is also specified, since $S_{0}(B)=0$, which requires that

$$
\left.\frac{d}{d E}\left(\frac{E \sigma}{\pi R^{2}}\right)\right|_{E=B}=\frac{1}{2} .
$$

With $S_{0}(E)$ known, Eq. (2b) can be inverted to obtain information about $V(r)$. This procedure is closely related to the solution of Abel's problem in classical mechanics ${ }^{12}$ and has been applied to one-dimensional barrier penetration by Cole and Good. ${ }^{13}$ We find that the distance between the inner and outer $L=0$ turning points (thickness of the barrier) at energy $V<B$ is given by

$$
\begin{aligned}
t(V) & =r_{2}(V)-r_{1}(V) \\
& =-\frac{2}{\pi}\left[\frac{\hbar^{2}}{2 m}\right]^{1 / 2} \int_{V}^{B} \frac{d S_{0} / d E}{\sqrt{E-V}} d E .
\end{aligned}
$$

As expected from the semiclassical nature of our treatment, Eq. (7) shows that $t(V)$ is determined only by $\sigma(E)$ for $V<E<B$.

To complete our method, we must specify the radius $R(E)$ associated with the angular momentum dependence of $T_{L}(E)$. On physical grounds, we expect that $r_{1}(E)<R(E)<r_{2}(E)$. Since the nuclear contribution in the internucleus potential is expected to be short range, attractive, and changing rapidly near $r=R_{B}$, we may approximate $r_{1}(E) \approx R_{B}$ and

$$
r_{2}(E) \approx R_{C}(E)=\frac{Z_{1}, Z_{2} e^{2}}{E},
$$

where $R_{C}$ is the Coulomb turning point $\left(Z_{1}\right.$ and $Z_{2}$ are the atomic numbers of the nuclei). Thus, a plausible choice is

$$
R(E)=\eta R_{B}+(1-\eta) R_{C}(E)
$$

with $0 \leq \eta \leq 1$, independent of $E$.

The barrier peak position, $R_{B}$, may be taken from a physical phenomenological potential, such as the KNS potential, or determined approximately from the data as fol- lows. Near the top of the barrier, it is a reasonable approximation to describe the barrier as a parabola, in which case Eq. (4) may be integrated analytically to obtain the familiar result ${ }^{14}$

$$
E \sigma(E) \equiv f(E)=\frac{\pi R_{B}^{2}}{a} \ln \left(1+e^{\alpha(E-B)}\right),
$$

where $a$ is inversely proportional to the oscillator frequency of the parabola. In this parabolic approximation, $B$ is determined by the condition

$$
\left.\frac{f^{\prime \prime} f}{f^{\prime} f^{\prime}}\right|_{E=B}=\ln 2
$$

from which $R_{B}$ is determined using Eq. (6).

We have verified the accuracy of our inversion method by applying it to cross sections generated from phenomenological internuclear potentials for ${ }^{12} \mathrm{C}+{ }^{12} \mathrm{C}$ (the universal Woods-Saxon potential of Ref. 15) and ${ }^{64} \mathrm{Ni}+{ }^{64} \mathrm{Ni}$ (the KNS potential), which span the region for which we analyze experimental data. These potentials and the thickness functions, $t(E)$, reconstructed from the cross sections using Eqs. (5)-(7) are shown in Fig. 1, where the left-hand panel refers to the ${ }^{12} \mathrm{C}+{ }^{12} \mathrm{C}$ system and the right-hand panel pertains to the ${ }^{64} \mathrm{Ni}+{ }^{64} \mathrm{Ni}$ system. For the ${ }^{12} \mathrm{C}+{ }^{12} \mathrm{C}$ system, when the exact value of $R_{B}$ is used, the choice $\eta=0.5$ in Eq. (8) allows $t(V)$ to be reproduced to within $0.05 \mathrm{fm}$, except for $V$ very close to $B$, where there is a discrepancy because (6) underestimates the barrier height by $0.15 \mathrm{MeV}$. Similarly, for the ${ }^{64} \mathrm{Ni}+{ }^{64} \mathrm{Ni}$ test case the choice of $\eta=0.5$ results in a difference of less than $0.03 \mathrm{fm}$ except close to the barrier maximum. Figure 1 also demonstrates the relative insensitivity of the results to the choice of $R_{B}$. Except near the top of the barrier, variation of $R_{B}$ by $\pm 1 \mathrm{fm}$ introduces errors less than $0.05 \mathrm{fm}$ for the ${ }^{12} \mathrm{C}+{ }^{12} \mathrm{C}$ system and 0.04 fm for the ${ }^{64} \mathrm{Ni}+{ }^{64} \mathrm{Ni}$ system. We have therefore adopted $\eta=0.5$ in all our analyses below and expect that the combined errors arising from the selection of $R_{B}$ and $\eta$ will be less than $0.1 \mathrm{fm}$.

We have applied our method to the experimental data for the systems ${ }^{13} \mathrm{C}+{ }^{13} \mathrm{C}$ (Ref. 15), ${ }^{14} \mathrm{~N}+{ }^{14} \mathrm{~N}$ (Ref. 16), ${ }^{13} \mathrm{C}+{ }^{16} \mathrm{O}$ (Ref. 17), ${ }^{40} \mathrm{Ca}+{ }^{40} \mathrm{Ca}$ (Ref. 18 ), ${ }^{58} \mathrm{Ni}+{ }^{64} \mathrm{Ni}$, ${ }^{64} \mathrm{Ni}+{ }^{64} \mathrm{Ni}, \quad{ }^{58} \mathrm{Ni}+{ }^{74} \mathrm{Ge},{ }^{64} \mathrm{Ni}+{ }^{74} \mathrm{Ge}$ (Ref. 2), and ${ }^{74} \mathrm{Ge}+{ }^{74} \mathrm{Ge}$ (Ref. 19). In contrast to such systems as ${ }^{12} \mathrm{C}+{ }^{12} \mathrm{C}$ and ${ }^{16} \mathrm{O}+{ }^{16} \mathrm{O}$, the excitation functions show no structure, the presence of which would invalidate our simple barrier-penetration picture. Since Eqs. (5)-(7) require energy derivatives of $\sigma(E)$, for reasons of numerical stability we have represented the logarithm of $\sigma$ by a leastsquares-adjusted sixth-order polynomial in the energy. For data and test cases from $\mathrm{C}$ to $\mathrm{Ni}$, the total $\chi^{2}$ is essentially independent of polynomial order beyond sixth order and results are insensitive to the precise order used. Errors in the thickness function are evaluated using standard linear error analysis. Noting the explicit and implicit dependence on the cross sections,

$$
t(E)=t\left(R\left(\left\{\sigma_{i}\right\}\right), B\left(\left\{\sigma_{i}\right\}\right),\left\{\sigma_{i}\right\}, E\right),
$$

the variance in $t$ is given by 


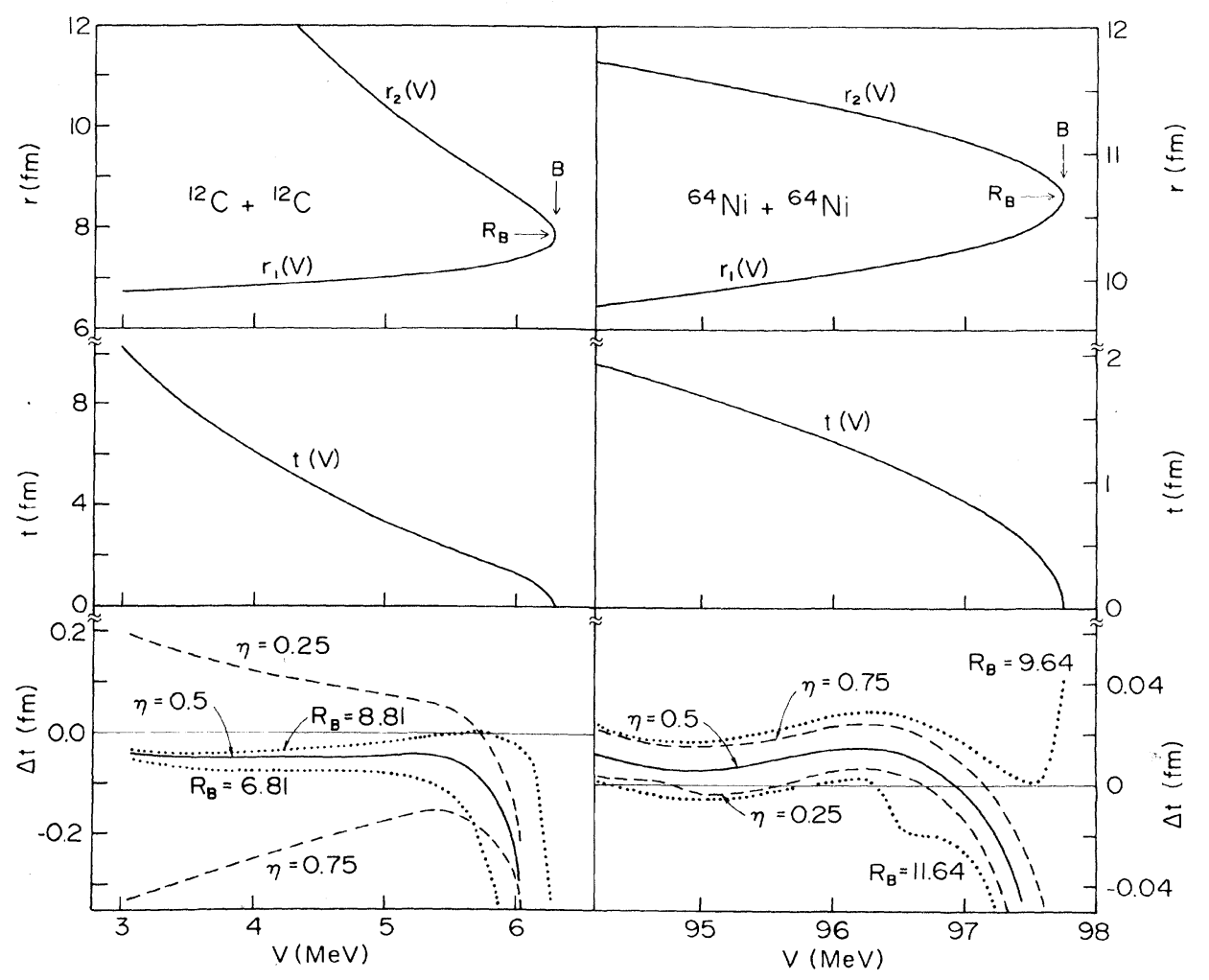

FIG. 1. Tests using model potentials for the ${ }^{12} \mathrm{C}+{ }^{12} \mathrm{C}$ system (left-hand panel) and the ${ }^{64} \mathrm{Ni}+{ }^{64} \mathrm{Ni}$ system (right-hand panel). Upper portion: Inner and outer barrier radii, $r_{1}(V)$ and $r_{2}(V)$, for two phenomenological potentials. The nuclear interaction for the ${ }^{12} \mathrm{C}+{ }^{12} \mathrm{C}$ system is the "universal" Woods-Saxon form for $p$-shell nuclei of Ref. 15. $V=-V_{0}\left\{1+\exp \left[\left(r-R_{0}\right) / a\right]\right\}^{-1}$ with $V_{0}=50$ $\mathrm{MeV}, R_{0}=r_{0}\left(A_{1}^{1 / 3}+A_{2}^{1 / 3}\right), r_{0}=1.27 \mathrm{fm}$, and $a=0.4 \mathrm{fm}$, where $A_{1}=A_{2}=12$ are the mass numbers of target and projectile. The nuclear interaction for the ${ }^{64} \mathrm{Ni}+{ }^{64} \mathrm{Ni}$ system is the "Yukawa plus exponential" KNS potential of Ref. 11, Eqs. (17)-(20). In both cases the Coulomb potential is that of two point charges. The exact barrier heights and positions $\left(B=6.30 \mathrm{MeV}, R_{B}=7.81 \mathrm{fm}\right.$ for ${ }^{12} \mathrm{C}+{ }^{12} \mathrm{C}$ and $B=97.74, R_{B}=10.64$ for $\left.{ }^{64} \mathrm{Ni}+{ }^{64} \mathrm{Ni}\right)$ are indicated. Middle portion: Barrier thickness, $t(V)=r_{2}(V)-r_{1}(V)$. Lower portion: Error in the thickness determined by the inversion procedure. The solid and dashed curves show the results for various $\eta$ when the exact value of $R_{B}$ is used. The dotted curves correspond to $\eta=0.5$ and the values of $R_{B}$ shown.

$$
\begin{aligned}
\left\langle(t-\langle t\rangle)^{2}\right\rangle=\sum_{i} & {\left[\frac{\partial t}{\partial R} \frac{\partial R}{\partial \sigma_{i}}+\frac{\partial t}{\partial B} \frac{\partial B}{\partial \sigma_{i}}+\frac{\partial t}{\partial \sigma_{i}}\right]^{2} } \\
& \times\left\langle\left(\sigma_{i}-\left\langle\sigma_{i}\right\rangle\right)^{2}\right\rangle,
\end{aligned}
$$

where the variance in $\sigma_{i}$ is taken to be the quoted experimental error. The error bands specified by Eq. (9) were checked in several cases by generating up to 40 sets of pseudodata in which data were perturbed by random errors of the proper variance and then inverted using Eq. (7). As will be seen subsequently in Fig. 2, typical error bands in the thickness functions inverted from experimental data are 0.1 to $0.2 \mathrm{fm}$, so the ambiguities arising from the choice $\eta=0.5$ or up to $1 \mathrm{fm}$ uncertainty in $R_{B}$ are unimportant. Uncertainties in $t$ are largest for $V \ll B$ (where the data are less precisely determined) and for $V \approx B$ (where $|\partial t / \partial V|$ is large).

In order to show how experimental errors in the fusion cross section are reflected in the thickness function we have used the ${ }^{13} \mathrm{C}+{ }^{13} \mathrm{C}$ system studied by Trentalonge et al. ${ }^{15}$ The experimental fusion cross sections are shown in the upper portion of Fig. 2. The barrier thickness determined by our inversion procedure using $R_{B}=7.8 \mathrm{fm}$ is shown in the lower portion of Fig. 2, together with the barrier height found from Eq. (6), $B=6.08 \pm 0.15 \mathrm{MeV}$.

Although the thickness function is essentially model independent within experimental errors, a slightly more model dependent but physically illuminating quantity is the local one-dimensional potential barrier itself. If the outer turning point is specified by physical arguments, the inner barrier may then be determined from the thickness function. Certainly well below the barrier, the outer turning point is unambiguously determined by the Coulomb potential. Up to the peak of the barrier, the nuclear corrections to the pure Coulomb potential are sufficiently weak that a phenomenological potential such as the KNS potential should yield an adequate representation of the outer turning point. In our analysis, we have taken $R_{B}$ to be the position of the peak of the KNS potential. [As emphasized above, the inversion formula is sufficiently insensitive to $R_{B}$ that this does not affect $t(V)$ within experimental errors.]. Since through Eq. (6) $R_{B}$ and the data uniquely determine $B$, the two coefficients of the KNS potential were slightly readjusted for each system to keep $R_{B}$ fixed but exactly reproduce this barrier height $B$. In the 


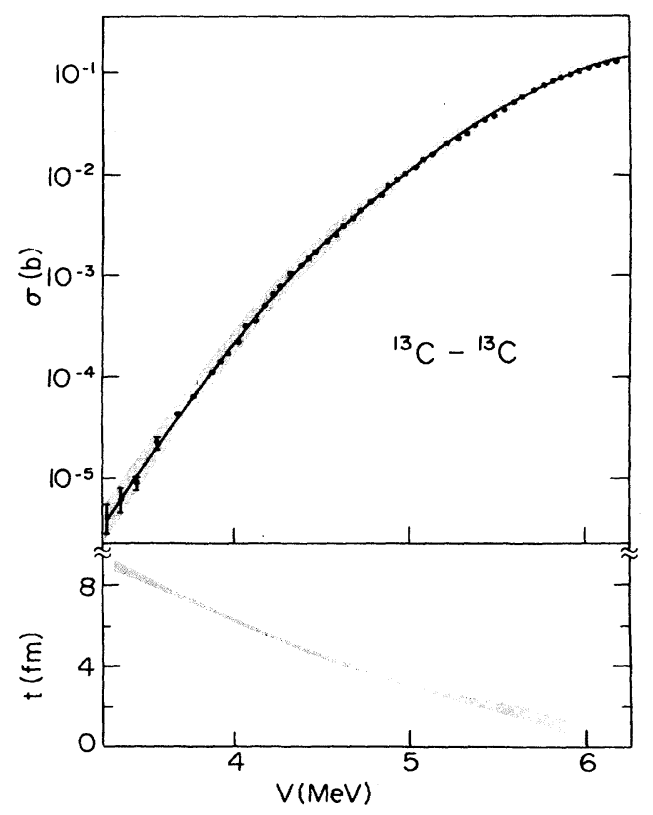

FIG. 2. Inversion of ${ }^{13} \mathrm{C}+{ }^{13} \mathrm{C}$ data. Upper portion: The experimental fusion excitation function from Ref. 14 is denoted by solid dots and error bars indicate statistical errors only. The dashed line denotes the sixth-order polynomial fit and the shaded region indicates the $15 \%$ systematic uncertainty in the data. Lower portion: The shaded region denotes the uncertainty in the thickness function $t(V)$ determined from the cross sections in the upper curve.

lighter nuclei, the shift was of the order of $0.2 \mathrm{MeV}$ and in heavy nuclei the shift was of the order $2 \mathrm{MeV}$. For each system, the effective potential was then determined by combining the experimental thickness function with this semiphenomenological outer turning point.

The resulting potentials for six typical systems are shown in Fig. 3. For comparison, point Coulomb and unmodified KNS potentials for these systems are also shown. Note that the experimentally determined quantity is the distance between the inner and outer turning points, $t(V)$, and that the error envelope denotes only the error in $t(V)$ and does not include the error in determining the outer turning point.

The three potentials determined by inversion of the data for light systems, ${ }^{13} \mathrm{C}+{ }^{13} \mathrm{C},{ }^{14} \mathrm{~N}+{ }^{14} \mathrm{~N}$, and ${ }^{13} \mathrm{C}+{ }^{16} \mathrm{O}$, are all qualitatively similar to the phenomenological KNS potentials and the deviation from the Coulomb potential is sufficiently small that the outer barrier is unambiguous. Quantitatively, the barriers are slightly thinner and the inner turning point curves are somewhat steeper than the phenomenological potential. The ${ }^{40} \mathrm{Ca}+{ }^{40} \mathrm{Ca}$ potential is much narrower than those arising in light nuclei and the interior turning point is almost vertical. The most striking feature of Fig. 3 is the fact that the ${ }^{64} \mathrm{Ni}+{ }^{64} \mathrm{Ni}$ and ${ }^{64} \mathrm{Ni}+{ }^{74} \mathrm{Ge}$ thickness functions are inconsistent with the assumption of a single-valued one-dimensional local potential, and similar results are obtained for ${ }^{58} \mathrm{Ni}+{ }^{64} \mathrm{Ni}$ and ${ }^{74} \mathrm{Ge}+{ }^{74} \mathrm{Ge}$.

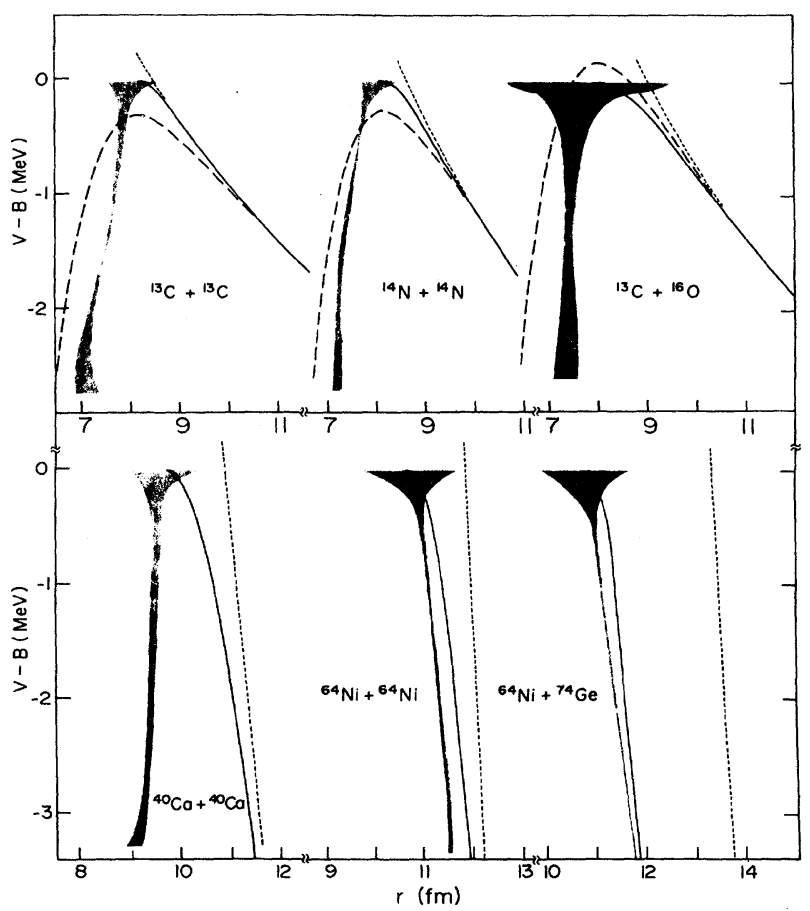

FIG. 3. Effective one-dimensional potential barriers for ${ }^{13} \mathrm{C}+{ }^{13} \mathrm{C},{ }^{14} \mathrm{~N}+{ }^{14} \mathrm{~N},{ }^{13} \mathrm{C}+{ }^{16} \mathrm{O},{ }^{40} \mathrm{Ca}+{ }^{40} \mathrm{Ca},{ }^{64} \mathrm{Ni}+{ }^{64} \mathrm{Ni}$, and ${ }^{64} \mathrm{Ni}+{ }^{74} \mathrm{Ge}$. The outer turning point is determined from the KNS potential readjusted as described in the text to fit the peak positions $\left[r_{B}(\mathrm{fm}), B(\mathrm{MeV})\right]=(8.11,6.13),(8.06,8.23),(8.16$, $7.52),(9.51,52.85),(10.64,95.77)$, and $(10.84,110.76)$ for these systems, respectively. The distance between the outer and inner turning points is the thickness function $t(V)$ inverted from the fusion cross sections and the shaded region indicates the error envelope specified by Eq. (9). The short dashed line denotes the point Coulomb potential, the long dashed curves in the upper figures denote the unmodified KNS potential, and for ease of presentation the energy is plotted relative to the barrier height $B$.

This analysis thus directly exposes the inadequacy of the assumption of a local, one-dimensional real potential with absorption into the fusion channel restricted to the region inside the barrier. A more general one-dimensional potential theory would utilize a complex local optical potential which allows absorption under the barrier, as well as inside the barrier. From purely geometrical considerations, it is evident that absorption under the barrier becomes more significant with increasing $A$, so it is understandable that the present description becomes invalid for sufficiently massive ions. Quantitatively, it is instructive to consider the distance between the top of the barrier $R_{B}$ and the position, $R_{0}$, of the surface of the Woods-Saxon potential of Ref. 15. For ${ }^{12} \mathrm{C}+{ }^{12} \mathrm{C}, R_{B}-R_{0}=2.0 \mathrm{fm}$, and the absorption is therefore expected to be well inside the barrier. In contrast, for ${ }^{40} \mathrm{Ca}+{ }^{40} \mathrm{Ca}, R_{B}-R_{0}=1.2$ fm and for ${ }^{64} \mathrm{Ni}+{ }^{64} \mathrm{Ni}, R_{B}-R_{0}=1.0 \mathrm{fm}$. Thus, appreciable absorption may be expected under the barrier and it is not surprising that the potentials derived in this work ignoring this absorption become unphysical for these nuclei. A more microscopic description would replace this com- 
plex one-dimensional potential by the appropriate physical effects, and this present analysis thus motivates further investigation of the role of collective quantum fluctuations of the nuclear surface and multidimensional dynamics of the fusion process.

The authors are grateful to Philip Siemens for his hospitality at North Zulch, Texas, where this collaborative ef- fect was initiated. J. W. Negele gratefully acknowledges fellowship support by the John Simon Guggenheim Memorial Foundation. This work was supported in part through funds provided by the U.S. Department of Energy (DOE) under Contract DE-AC02-76 ERO3069 and the National Science Foundation under Grants PHY79-23638 and PHY 82-07332.
${ }^{1}$ L. C. Vaz, J. M. Alexander, and G. R. Satchler, Phys. Rep. C69, 373 (1981).

${ }^{2}$ M. Beckerman, M. Salomaa, S. Sperduto, J. D. Molitaris, and A. DiRienzo, Phys. Rev. C 25 , 837 (1982).

${ }^{3}$ C. A. Barnes, in Treatise on Heavy-Ion Physics, edited by D. A. Bromley (Plenum, New York, in press).

${ }^{4}$ P. Descouvemont, O. Baye, and P. H. Heenen, Z. Phys. A $\underline{306}$, 79 (1982).

${ }^{5}$ M. J. Rhoades-Brown and V. E. Oberacker, Phys. Rev. Lett. 50, 1435 (1983).

${ }^{6}$ H. Esbensen, Nucl. Phys. A352, 147 (1981).

${ }^{7}$ W. Reisdorf et al., Phys. Rev. Lett. 49, 1811 (1982).

${ }^{8}$ S. Landowne and J. R. Nix, Nucl. Phys. A368, 352 (1981).

${ }^{9}$ L. D. Landau and E. M. Lifshitz, Quantum Mechanics: NonRelativistic Theory (Pergamon, New York, 1965).

10D. L. Hill and J. A. Wheeler, Phys. Rev. 9, 1102 (1953).

${ }^{11}$ H. J. Krappe, J. R. Nix, and A. J. Sierk, Phys. Rev. C 20, 992
(1979).

${ }^{12}$ L. D. Landau and E. M. Lifshitz, Mechanics (Pergamon, New York, 1969).

${ }^{13}$ M. W. Cole and R. H. Good, Phys. Rev. A 18 , 1085 (1978).

${ }^{14}$ C. Y. Wong, Phys. Rev. Lett. 31, 766 (1973).

${ }^{15} \mathrm{~S}$. Trentalonge, Ph.D. dissertation, California Institute of Technology, 1982 (unpublished).

${ }^{16}$ Z. E. Switkowski, R. G. Stokstad, and R. M. Wieland, Nucl. Phys. A274, 202 (1976).

${ }^{17}$ B. Dasmahapatra, B. Cujec, and F. Lahlou, Nucl. Phys. A394, 301 (1983).

${ }^{18}$ H. Al-Juwair, R. Ledoux, M. Beckerman, E. Cosman, S. Gazes, M. Salomaa, J. Wiggins, R. Betts, S. Saini, and O. Hansen, Bull. Am. Phys. Soc. 28, 670 (1983); and to be published.

${ }^{19}$ M. Beckerman, M. K. Salomaa, J. Wiggins, and R. Rohe (unpublished). 